\title{
P-selectin-mediated platelet activation promotes adhesion of non-small cell lung carcinoma cells on vascular endothelial cells under flow
}

\author{
LIANG GONG $^{1}$, HONG-JIAN MI ${ }^{2}$, HONGCHU ZHU ${ }^{2}$, XIANGDONG ZHOU ${ }^{1}$ and HEPING YANG ${ }^{1}$ \\ ${ }^{1}$ Department of Respiration, The Southwest Hospital; ${ }^{2}$ Teaching and Research Section of Anthropotomy, \\ The Third Military Medical University, Chongqing 400038, P.R. China
}

Received October 8, 2011; Accepted January 3, 2012

DOI: $10.3892 / \mathrm{mmr} .2012 .760$

\begin{abstract}
Lung cancer is a severe disease threatening human health worldwide. Distant hematogenous metastasis results in poor prognosis and death of lung cancer patients. In the present study, we investigated the effect of circulatory platelets (PLTs) on hematogenous metastasis of non-small cell lung carcinoma (NSCLC). Laser scanning confocal microscopy was employed to assay the expression of P-selectin in lung cancer tissue, paracancerous tissue and distant tissue, respectively. Meanwhile, fluorescence-activated cell sorting (FACS) was used to determine P-selectin activation in peripheral blood. Purified PLTs were co-cultured with A549 cells and human vascular endothelial cells (HuvECs). Subsequently, the formation of PLT-lung cancer cell complexes and their effects on rolling and adhesion of A549 on the surface of vascular endothelium were assayed. Integrin $\alpha 3, \alpha 5, \beta 1$, ICAM-1 and VCAM-1 mRNAs and proteins were measured by reverse RT-PCR and western blot analysis, respectively. The expression of P-selectin in lung adenocarcinoma tissue was significantly stronger compared to that in paracancerous and distant tissues. P-selectin activation in peripheral blood in lung adenocarcinoma was markedly enhanced. The rolling rate of A549 on HuvECs was significantly slowed down after co-culture of activated PLTs and A549 cells. The mRNA and protein levels of integrin $\alpha 3, \alpha 5, \beta 1$, ICAM- 1 and VCAM-1 were significantly increased after the co-culture. In conclusion, the PLT-lung cancer cell complexes protected the lung cancer cells from mechanical injury under blood flow. Furthermore, up-regulated integrin $\alpha 3, \alpha 5, \beta 1$ and endothelial cell adhesion molecules ICAM-1 and VCAM-1 promoted the adhesion of A549 on vascular endothelial cells, which may be responsible for hematogenous metastasis of lung cancer.
\end{abstract}

Correspondence to: Dr Heping Yang, Department of Respiration, The Southwest Hospital, The Third Military Medical University, Gaotanyan Main Street No. 30, Shapingba, Chongqing 400038, P.R. China

E-mail: mkojima@163.com

Key words: lung cancer, hematogenous metastasis, platelet activation, P-selectin, adhesion

\section{Introduction}

Lung cancer is the leading cause of cancer-related deaths, and $80 \%$ of lung cancer cases are non-small cell lung cancer (NSCLC). Distant hematogenous metastasis is one of the main reasons for poor prognosis of lung cancer patients $(1,2)$. In the blood circulation, lung cancer cells interact with a variety of cellular components and cytokines, which affect the hematogenous metastasis of cancer cells. In fact, platelet (PLT) activation may play an important role in this event (3).

In the present study we first investigated the blood PLT count in peripheral blood and the expression of platelet P-selectin in patients with NSCLC and then compared these values with a healthy control population. Purified PLTs were then co-cultured with lung adenocarcinoma cell line A549 and human vascular endothelial cells (HuvECs). A flow chamber simulation assay was subsequently performed to investigate the formation of PLT-lung cancer cell complexes and the effects on rolling and adhesion of A549 calls on the surface of vascular endothelium. Finally, the possible mechanism was further studied. Based on this, we expected to elucidate the correlation between PLT activation and hematogenous metastasis of lung cancer.

\section{Materials and methods}

\section{Materials}

Clinical data. One hundred and sixty-eight cases of primary lung cancer excluding patients with hematological systemic diseases, severe malnutrition, infection, cardiovascular diseases and diabetes were enrolled in the present study after preliminary diagnosis at the Department of Respiration of The Southwest Hospital from June 2008 to August 2009 (Chongqing, China). The mean patient age was 53 \pm 19 years. All of the cases were confirmed by pathological examination, including 53 cases of squamous cell carcinoma, 44 cases of NSCLC and 7 cases of other pathological types such as largecell carcinoma and carcinoid tumors.

TNM staging for lung cancer was performed in accordance with the standards of AJCC and UICC. All of the patients did not receive any drugs which might affect the function of the platelets 2 week prior to the blood sample collection. Thirty 
healthy volunteers including 19 females and 11 males were selected as the control and their mean age was $44 \pm 9$ years. In the present study, all of the subjects read and signed informed consent forms. This study was approved by the Ethics Committee of The Southwest Hospital.

Cell line culture. A549 cells and HuvECs were purchased from the Shanghai Cell Repository (Shanghai, China). A549 cells were routinely cultured in Dulbecco's modified Eagle's medium (DMEM) (Hyclone, USA) containing 10\% fetal calf serum (Gibco, USA). HuvECs were cultured in M200 medium (Hyclone) containing 20\% fetal calf serum and $2 \mathrm{mmol} / \mathrm{l}$ L-glutaminate (Sigma, USA). The cells were cultured in an atmosphere of $5 \% \mathrm{CO}_{2}$ at $37^{\circ} \mathrm{C}$ and passaged every 2-3 days.

Main reagents. Mouse anti-human integrin $\alpha 3$, integrin $\alpha 5$, integrin $\beta 1$, ICAM- 1 and VCAM-1 antibodies were provided by Santa Cruz Biotechnology, Ltd. (Santa Cruz, CA, USA). RNA Plus isolation and RT-PCR kits were products of Takara Bio., Ltd. (Osaka, Japan). Lipofectamine ${ }^{\mathrm{TM}} 2000$ was obtained from Invitrogen Co. (Shanghai, China). pSilencer 2.0-U6 plasmid carrying GFP was purchased from the GenScript Corp., as well as the RNAi-negative control, the pSilencer-PSGL1-NON RNAi plasmid.

Expression of P-selection in lung cancer tissues of different pathological types. Laser scanning confocal microscopy (LSCM) manipulation was performed as follows. i) Lung cancer tissue, paracancerous tissue and distant tissue were removed and washed with PBS three times and then cut into a size of $\sim 0.3 \times 0.5 \mathrm{~cm}$. The samples were consecutively cut into $5-\mu \mathrm{m}$ sections. Sections were fixed with cold acetone for $10 \mathrm{~min}$ and washed with $0.1 \mathrm{M}$ PBS three times each for $4 \mathrm{~min}$. ii) Respectively, mouse anti-human integrin $\alpha 3$, integrin $\alpha 5$, integrin $\beta 1$, ICAM-1 or VCAM-1 antibody (1:200) was added to the sections overnight at $4^{\circ} \mathrm{C}$ in a humidity chamber. iii) The sections were washed with $0.1 \mathrm{M}$ PBS three times each for 4 min. iv) Fluorescence-labeled rabbit anti-mouse $\operatorname{IgG}(1: 3000)$ was added and co-cultured for $30 \mathrm{~min}$ at $37^{\circ} \mathrm{C}$ and then washed with $0.1 \mathrm{M}$ PBS three times each for $4 \mathrm{~min}$. v) 4,6-Diamidino-2-phenylindole (DAPI) was used to stain the nuclei for $10-20 \mathrm{~min}$ at $37^{\circ} \mathrm{C}$ and then rinsed with $0.1 \mathrm{M} \mathrm{PBS}$ for three times each for $4 \mathrm{~min}$. vi) The sections were mounted with $70 \%$ glycerine (v/v) followed by LSCM assay.

PLT isolation. PLT isolation was performed as follows. i) Blood samples were collected from the subjects via vein in the morning. Anticoagulated whole blood (7 ml) was collected and centrifuged at $2500 \mathrm{rpm}$ for $12 \mathrm{~min}$ at room temperature. ii) The supernatants were harvest and then centrifuged at $3200 \mathrm{rpm}$ for $30 \mathrm{~min}$ at $4^{\circ} \mathrm{C}$. The supernatants were extracted and PLTs were collected. iii) The collected PLTs were washed with $0.01 \mathrm{M} \mathrm{PBS}$ and then centrifuged at $3200 \mathrm{rpm}$ for $30 \mathrm{~min}$ at $4^{\circ} \mathrm{C}$.

Blood PLT count (BPC) assay. Based on the BPC in the peripheral blood prior to chemotherapy, radiotherapy or surgery, BPC was classified into decreased $\left(<100 \times 10^{9} / 1\right)$,

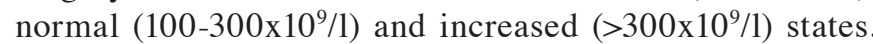
Meanwhile, the correlations between the change in peripheral
PLTs and tumor pathological type and metastasis stage in the groups were compared and analyzed.

FACS assay of the expression of P-selectin in peripheral blood. After isolation and purification, the PLTs were re-suspended in $50 \mu \mathrm{l}$ of PBS $(0.1 \mathrm{M})$ followed by the addition of $5 \mu \mathrm{l}$ of P-selectin antibody (1:50). Then the PLTs were incubated at $37^{\circ} \mathrm{C}$ for $1 \mathrm{~h}$. The PLTs were washed with PBS $(0.1 \mathrm{M})$ and centrifuged at $3000 \mathrm{rpm}$ for $3.5 \mathrm{~min}$. The process was repeated. Subsequently, $5 \mu$ l of FITC-labeled secondary antibody (1:50) was added and incubation was carried out at $37^{\circ} \mathrm{C}$ for $1 \mathrm{~h}$. The PLTs were washed with PBS (0.1 M) twice and measured using a Cell Lab Quanta SC Coulter flow cytometer(Beckman Coulter, USA).

Small interference RNA (siRNA) targeting PSGL1 and transfection. According to the PSGL-1 gene sequence in GenBank, the specific siRNA sequence for PSGL-1 (GI: 68160948) available at http://www.ncbi.nlm.nih.gov/nuccore/NM_003006.3 was designed by using an siRNA design tool 'siRNA sequence selector'. Then three sequences including 5'-CCACGGATTCA GCAGCTAT-3', 5'-GGAGATACAGACCACTCAA-3', 5'-GGA AGCACAGACCACTCAA-3' were selected. After the primary experiment, the sequence 5'-GGAGATACAGACCACT CAA-3' with best blockage was used for the following transfection.

The construction of the pSilencer-PSGL1-shRNA vector was completed by Shanghai GeneChem Co., Ltd. and then the manipulation was performed as follows. i) The oligonucleotide fragments were annealed to form double-strand structure. ii) pSilencer-PSGL1-shRNA was digested with restriction enzymes to retrieve the line empty vector. iii) The target fragments were connected with the linearized empty vectors. iv) The plasmid was transfected into fresh E.coli $\mathrm{DH} 5 \alpha$ and screened on an LB plate containing ampicillin $(100 \mu \mathrm{g} / \mathrm{ml})$ and the positive colonies were picked for sequence identification (Takara Corp., Dalian, China).

A day before transfection, $0.5-2 \times 10^{5}$ tumor cells were inoculated into 6-well plates. The pSilencer-PSGL1-shRNA plasmids were transfected into the A549 cells according to the methods described in the instructions for Lipofectamine ${ }^{\mathrm{TM}}$ 2000. The culture plates were incubated at $37^{\circ} \mathrm{C}$ in an incubator with $5 \% \mathrm{CO}_{2}$. After a 48-h transfection, the cells were collected to perform the subsequent FACS operations. The cells after a 48-h transfection were digested and removed from the plates and were washed twice with pre-cooled PBS and then centrifuged at $1500 \mathrm{rpm}$ for $5 \mathrm{~min}$. The cells were re-suspended and adjusted to a concentration of $20 \times 10^{6} / 1$. They were selected by FACS to obtain GFP-positive cells; the screening process was sterile and double-antibiotics (penicillin and streptomycin) were allowed to be added if necessary.

Assay of PLT adhesion under a scanning electron microscope (SEM). The cells were co-cultured as follows: i) A549, ii) A549 + inactivated PLTs, iii) A549 + activated PLTs, iv) A549 (siRNA targeting PSGL-1) + activated PLTs, v)A549 + activated PLTs + P-selectin antibody.

After the co-culture, the cells were fixed overnight at $4{ }^{\circ} \mathrm{C}$ and washed with $0.9 \% \mathrm{NS}$ twice each for $10 \mathrm{~min}$. Then the specimens were dehydrated using 30, 50, 70, 80, 90 and $100 \%$ 
Table I. Primer pairs for RT-PCR.

\begin{tabular}{llc}
\hline Gene & \multicolumn{1}{c}{ Primer pairs } & Product size (bp) \\
\hline Integrin $\alpha 3$ & PF: 5'-CACTCTGCTGGTGGACTATACACT-3' & 135 \\
& PR: 5'-TACTTGAGGGGGCTTCCTACAT-3' \\
Integrin $\alpha 5$ & PF: 5'-CTGTGACTACTTTGCCGTGAAC-3' & 110 \\
PR: 5'-GATGAGGGACTGTAAACCGAAG-3' & PF: 5'-CGTAGCAAAGGAACAGCAGAG-3' \\
Integrin $\beta 1$ & PR: 5'-GGTCAATGGGATAGTCTTCAGC-3' & 142 \\
ICAM-1 & PF: 5'-GTCACCTATGGCAACGACTCCT-3' \\
VCAM-1 & PR: 5'-AGTGTCTCCTGGCTCTGGTTC-3' & 119 \\
$\beta$-actin & PF: 5'-CCGATCACAGTCAAGTGTTCAG-3' & \\
& PR: 5'-CTCTTGGTTTCCAGGGACTTC-3' & \\
& PF: 5'-TTCTACAATGAGCTGCGTGTG-3'
\end{tabular}

PF, forward primer; PR, reverse primer.

alcohol, respectively, 4 min for each step. Then the specimens were administered 50, 70, 90, 95 and 100\% tert butyl alcohol, respectively $4 \mathrm{~min}$ for each step. Finally, the specimens were observed using an S-3400N SEM (Hitachi, Japan).

Assay of tumor cell adhesion. The temperature in the flow chamber was maintained at $37^{\circ} \mathrm{C}$, and the cells were placed in an atmosphere of mixed gases containing $95 \% \mathrm{O}_{2}$ and $5 \% \mathrm{CO}_{2}$. This system produces stable laminar shear stress ranging from 0 to $48 \mathrm{dyn} / \mathrm{cm}^{2}$. It simulates different blood flow conditions after the regulation. Previously, flow rates $(\mathrm{Q}, 0.5 \mathrm{ml} / \mathrm{min} ; \tau$, $\left.9.75 \mathrm{dyn} / \mathrm{cm}^{2}\right),\left(\mathrm{Q}, 0.3 \mathrm{ml} / \mathrm{min} ; \tau, 5.85 \mathrm{dyn} / \mathrm{cm}^{2}\right)$ and $(\mathrm{Q}, 0.1 \mathrm{ml} /$ $\min ; \tau, 1.95 \mathrm{dyn} / \mathrm{cm}^{2}$ ) were used to monitor the rolling and adherence of lung cancer cells on the surface of the endothelial cells.

Based on the previous study, we selected $0.1 \mathrm{ml} / \mathrm{min}$ as an appropriate flow rate. After the co-culture of PLTs and lung cancer cells, the cells were co-cultured again as following: i) HuvEC+A549; ii) HuvEC+A549 + inactivated PLTs; iii) HuvEC+A549 (siRNA targeting PSGL-1) + activated PLTs; iv) HuvEC+A549 + activated PLTs + P-selectin antibody.

HuvECs $\left(1 \times 10^{6} / \mathrm{ml}\right)$ were inoculated on coverslips in 6-well plates. HuvECs were co-cultured with PLTs and A549 for $24 \mathrm{~h}$. After that, the coverslips were placed in the flow chamber. The perfusate was prepared and the flow rate was adjusted. The treated PLTs and A549 cells were injected into the flow chamber. The rolling time and adhesion of A549 on vascular endothelium were observed and recorded along with the perfusate flow. The rolling and adhesion of A549 cells on the surface of the vascular endothelium and PLT aggregation were observed and recorded using an inverted fluorescence microscope in the absence or presence of a variety of flow rates. Meanwhile, a video was used to record the time required for the lung cancer cells to pass through per visual field unit.

RT-PCR assay of $m R N A$ expression of integrin $\alpha 3$, integrin $\alpha 5$, integrin $\beta 1, I C A M-1$ and VCAM-1. The cells were collected using centrifugation of $1000 \mathrm{rpm}$ for $10 \mathrm{~min}$. Then total RNA was prepared in accordance with the manufacturer's instructions. After DNase I treatment, $2 \mu \mathrm{g}$ of RNA was reverse transcribed with AMV reverse transcriptase. A master mix containing the reaction buffer, dNTPs, Taq polymerase and $2 \mu \mathrm{l}$ cDNA in a $25-\mu 1$ reaction mixture was transferred to different PCR tubes. Forward and reverse primers corresponding to different individual genes were added to the PCR tubes and subjected to PCR amplification using primers against various genes.

These reactions were performed for 30 cycles. The annealing temperature was maintained at $56^{\circ} \mathrm{C}$; the resting conditions included denaturation at $94^{\circ} \mathrm{C}$ for $30 \mathrm{sec}$ followed by extension at $72^{\circ} \mathrm{C}$ for $45 \mathrm{sec}$. The PCR products were determined using $1.5 \%$ agarose gel electrophoresis and ethidium bromide staining. Images of the gels were analyzed using the Quantity One software (Bio-Rad), which compares the relative density between objective straps and $\beta$-actin. The primers pairs are listed in Table I.

Western blot assay of protein expression of integrin $\alpha 3$, integrin $\alpha 5$, integrin $\beta 1, I C A M-1$ and VCAM-1 proteins. The tissue samples were dissolved in lysis buffer containing $7 \mathrm{~mol} / \mathrm{l}$ urea, $2 \mathrm{~mol} / \mathrm{l}$ thiourea and 4\% CHAPS (w/v). They were centrifuged at $40,000 \mathrm{x} \mathrm{g}$ for $1 \mathrm{~h}$ and then the supernatants were harvested. The total protein concentration was determined using the Bradford method.

Total protein $(25 \mu \mathrm{g})$ in each sample was separated by $12 \%$ SDS-PAGE. The proteins were transferred onto a sheet of polyvinylidene fluoride (PVDF) membrane with wet transfer $100 \mathrm{~V}$ for $1.5 \mathrm{~h}$. The membrane was probed with primary mouse anti-human integrin $\alpha 3$, integrin $\alpha 5$, integrin $\beta 1$, ICAM-1 and VCAM-1 antibodies (1:1000) overnight at $4^{\circ} \mathrm{C}$. The membrane was washed three times with Tris-buffered saline (TBS) for $5 \mathrm{~min}$ each and secondary rabbit anti-mouse IgG (1:2500) (Zhongshan Golden Bridge, Beijing, China) was added. The membrane was washed once with TBS for another 


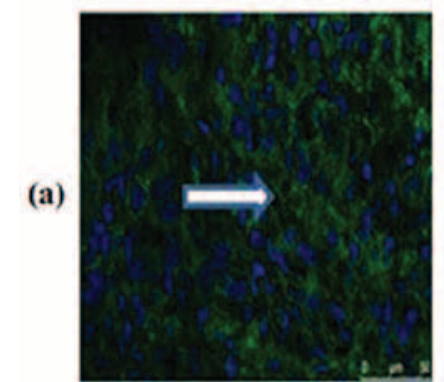

Lung adenocarcinoma

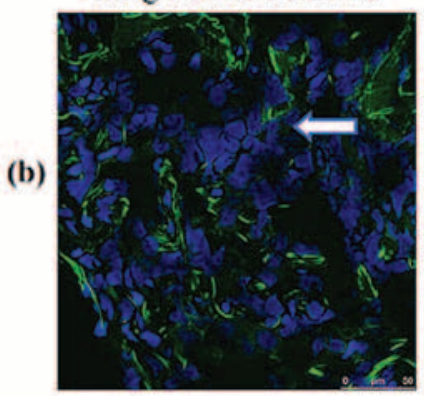

Lung squamous

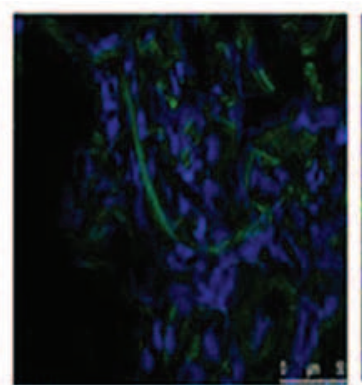

Paracancerous tissue

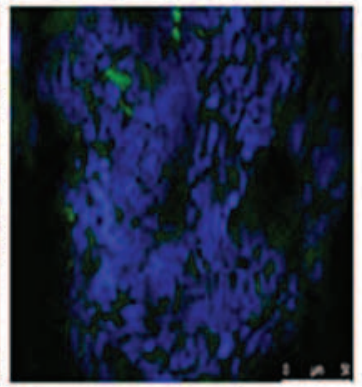

Paracancerous tissue

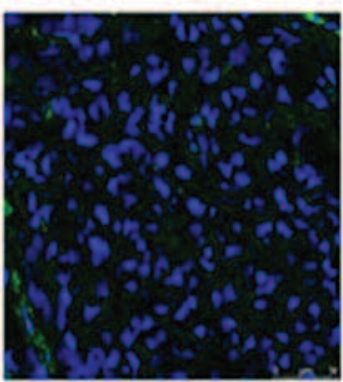

Distant tissue

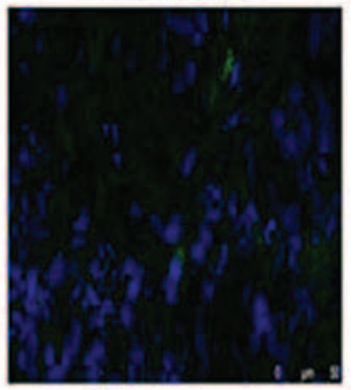

Distant tissue

Figure 1. Expression of P-selectin in different lung cancer tissue. Expression of P-selectin in lung adenocarcinoma tissue was stronger than that in lung squamous tissue; meanwhile, the expression of P-selectin in the cancer tissue was significantly increased compared with that in the paracancerous tissue and distant normal tissue, respectively.

5 min. Then it was stained by an enhanced chemiluminescence (ECL) reagent (Pierce, USA) and imaged on X-ray film by autoradiography. $\beta$-actin was used as a control. The band intensity was measured by Quantity One software (Bio-Rad) and the relative proteins levels were expressed as the ratios between the densities of objective proteins and $\beta$-actin.

Statistics and presentation of data. All experiments were performed at least three times, and representative data were presented. SPSS 12.0 statistical software was used to analyze the differences. The differences among groups were analyzed using one-way ANOVA or $\chi^{2}$ test. A P-value of $<0.05$ was considered to denote statistical significance.

\section{Results}

Expression of P-selectin in the different lung tissues. Compared with lung squamous carcinoma, the expression of $\mathrm{P}$-selectin in lung adenocarcinoma tissue was significantly enhanced (Fig. 1); the expression of P-selectin in the cancer tissue was stronger than that in the paracancerous and distant normal tissues, respectively (Fig. 1).

Correlation between BPC in the peripheral blood and pathological types. The PLT count was decreased, was normal and was increased in $3.57 \%(6 / 168), 69.05 \%(116 / 168)$ and $27.38 \%$ (46/168) of the 168 lung cancer patients, respectively. The PLT was increased in $22.64 \%$ (12/53) of squamous cell carcinoma, $37.09 \%(23 / 62)$ of adenocarcinoma, $22.70 \%$ (10/44) of small cell carcinoma cases and $14.20 \%(1 / 7)$ of other pathological types, respectively (Fig. 2).

The mean BPC was $(233.85 \pm 102.23) \times 10^{9} / 1,(269.89 \pm 128.20)$ x10 $/ 1,(238.41 \pm 92.04) \times 10^{9} / 1$ in lung squamous cell carcinoma,

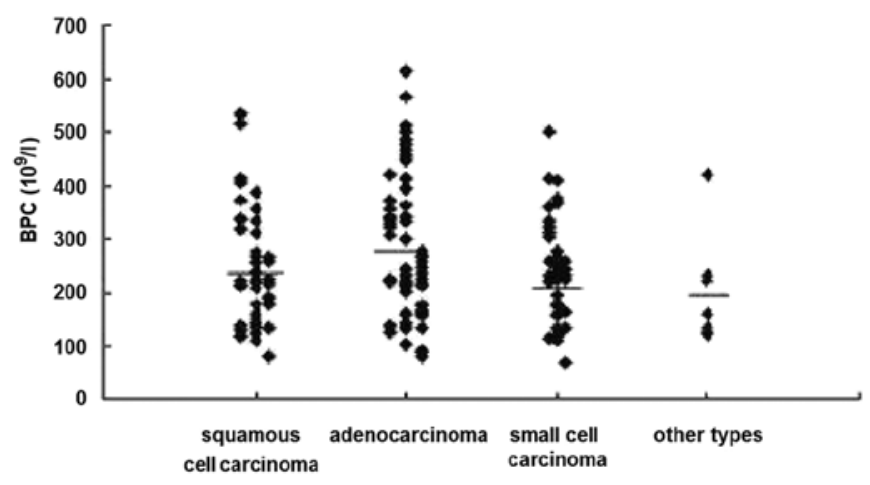

Figure 2. Correlation between blood platelet count (BPC) and pathological types in 168 patients with primary lung cancer based on the BPC in the peripheral blood prior to chemotherapy, radiotherapy or surgery. BPC was classified into decreased $\left(<100 \times 10^{9} / 1\right)$, normal $\left(100-300 \times 10^{9} / 1\right)$ and increased $\left(>300 \times 10^{9} / 1\right)$ states. We found that the BPC was decreased, was normal and was increased in $3.57 \%(6 / 168), 69.05 \%(116 / 168)$ and $27.38 \%(46 / 168)$ of the 168 lung cancer patients, respectively. Meanwhile, the PLT count was increased in $22.64 \%(12 / 53)$ of squamous cell carcinoma, in $37.09 \%(23 / 62)$ of adenocarcinoma, in $22.70 \%$ (10/44) of small-cell carcinoma and in $14.20 \%$ $(1 / 7)$ of other pathological types, respectively.

adenocarcinoma and small-cell carcinoma cases, respectively. Meanwhile, the BPC in the healthy control group was sustained at a normal level. Although no significant differences among groups were noted, there was an obvious increased tendency in $\mathrm{BPC}$ in the lung adenocarcinoma.

\section{Correlations between BPC and hematogenous metastasis and} clinical stage. Generally, hematogenous metastasis suggests poor prognosis and a clinical TNM stage of IV. Here, we analyzed the correlations between the change in BPC and TNM stage and hematogenous metastasis. 


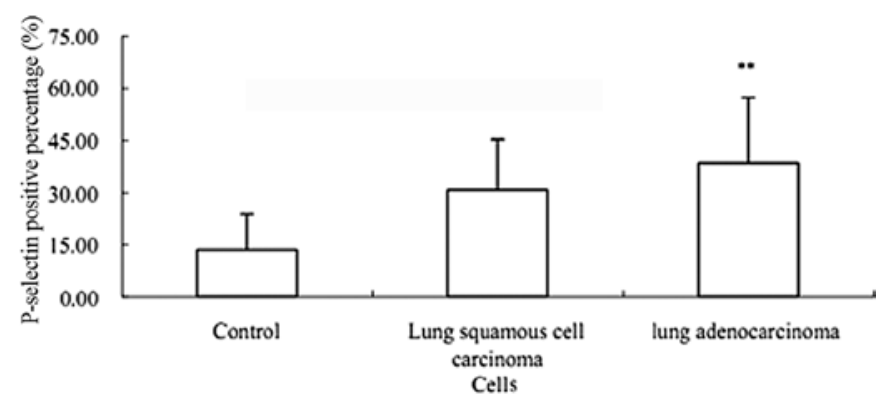

Figure 3. Activation of P-selectin in peripheral blood of NSCLC patients (mean $\pm \mathrm{SD}$ ). Expression of P-selectin in lung adenocarcinoma was significantly enhanced compared with that in the healthy control. However, there were no significant differences between squamous cell carcinoma and adenocarcinoma. ${ }^{* *} \mathrm{P}<0.01$ as compared with the control.

The results showed that the BPC was increased in $27.38 \%$ $(46 / 168)$ of the lung cancer patients. No increased BPC was found in stage I and II in the 46 patients. However, the BPC was increased in $19.35 \%$ of the patients in stage III. Furthermore, the BPC was significantly increased in $36.96 \%$ of the patients in stage IV (metastasis). Compared with stage I+II and stage III, there were significant differences $(\mathrm{P}<0.01)$ (Table II).

FACS assay of P-selectin in peripheral blood. The FACS results showed that the expression of PLT activation marker P-selectin in lung adenocarcinoma was significantly enhanced compared with that in the healthy control $(\mathrm{P}<0.01)$ (Fig. 3). However, there was no significant difference between adenocarcinoma and squamous cell carcinoma (P>0.05) (Fig. 3).

Construction and identification of siRNA targeting PSGL-1. The complementary double-strand PSGL-1 siRNA was directionally sub-cloned into pSilencer-GFP2.0 U6 eukaryotic expression vector by using DNA recombination technology.
Table II. Correlations between BPC and clinical stages.

\begin{tabular}{lrrrr}
\hline & \multicolumn{4}{c}{ Clinical stages (TNM) } \\
\cline { 2 - 5 } BPC & I+II & III & IV & Total \\
\hline$<100 \times 10^{9} / 1$ & 1 & 3 & 2 & 6 \\
$100-300 \times 10^{9} / 1$ & 13 & 47 & 56 & 116 \\
$>300 \times 10^{9} / 1$ & 0 & 12 & $34^{\mathrm{a}}$ & 46 \\
Total & 14 & 62 & 92 & 168
\end{tabular}

${ }^{*} \mathrm{P}<0.01$ vs. stage I+II and stage III. BPC, blood PLT count.

The pSilencer-PSGL1-shRNA eukaryotic expression plasmid was obtained. The strain for plasmid cloning was sent to the Takara Corp. for sequencing identification and the sequence results were identified.

SEM assay of PLT aggregation after co-culture. A small number of lung cancer cells assembled with PLTs in the control group. After RNAi targeting PSGL-1 in A549 cells, the interactions between activated PLTs and lung cancer cells were halted and little PLT aggregation was noted (Fig. 4A). Activated PLTs increased the interactions between platelets and cancer cells (Fig. 4B).

Adhesion of lung cancer cells on vascular endothelial cells under flow. Blood flow simulation assay showed that the co-culture of activated PLTs and A549 significantly attenuated the rolling rate of A549 on HuvECs, which promoted the interaction between A549 cells and HuvECs.

Compared with the control, the adhesion efficiency reached $65 \%$ and the rolling rate of A549 was decreased by $60 \%$ after the co-culture. However, the adhesion of A549 on HuvECs
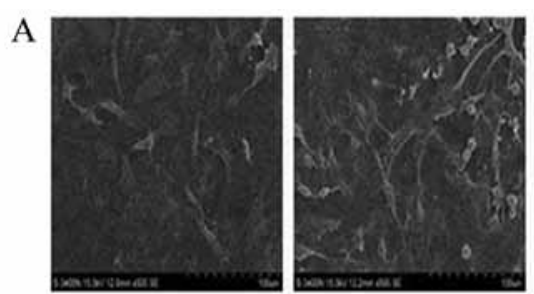

$1(\times 500)$

$2(\times 500)$

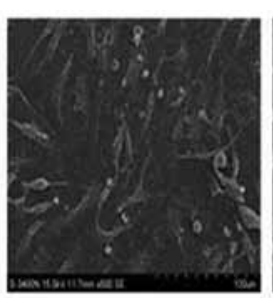

$3(\times 500)$

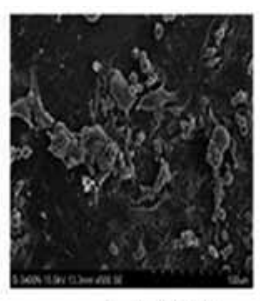

$4(\times 500)$

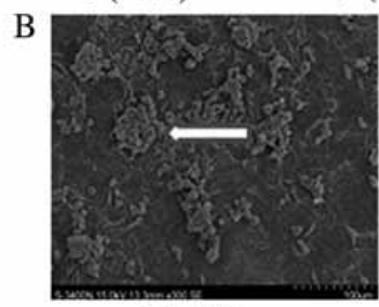

$1(\times 300)$

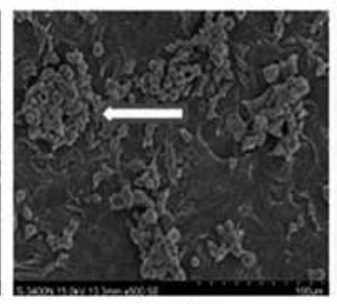

$2(\times 500)$

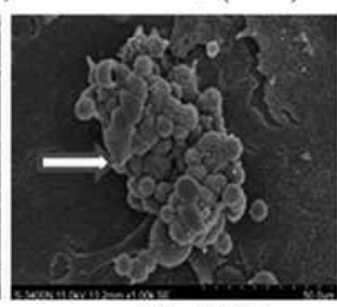

$3(\times 1000)$

Figure 4. SEM assay of PLT adhesion. Few lung cancer cells assembled with PLTs in the control group. (A) After siRNA targeting PSGL-1 or usage of specific P-selectin antibody in A549, little PLT aggregation was noted. (B) However, activated PLTs promoted interactions between cancer cells and endothelial cells and obvious aggregation phenomenon was observed. A: 1, A549; 2, A549 + inactive PLTs; 3, A549 (siRNA targeting PSGL-1) + activated PLTs; 4, A549 + activated PLTs + P-selectin antibody. (B) A549 + activated PLTs. The arrows in the figures represented PLT-lung cancer cell complexes. 

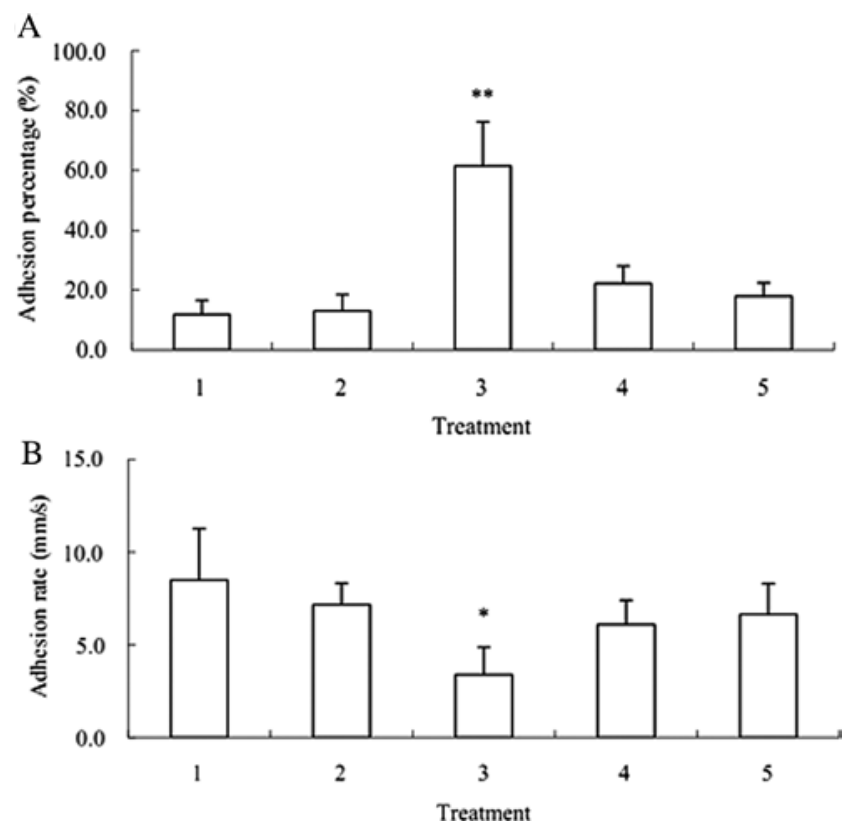

Figure 5. Adhesion of A549 cells on the surface of HuvECs compared with the control. The adhesion efficiency reached 65\% and the rolling rate of A549 was decreased by $60 \%$ after co-culture. There were significant differences between the two co-cultures. This suggests that the co-culture of activated PLTs and A549 cells significantly attenuated the rolling rate of A549 on HuvECs, which promoted the interaction between A549 and HuvECs. (A) Adhesion between endothelial cells and lung adenocarcinoma cells after interaction with PLTs. (B) Rolling rate of lung adenocarcinoma cells. 1, HuvECs+A549; 2, HuvECs+A549 + inactivated PLTs; 3, HuvECs+A549 + activated PLTs; 4, HuvECs+A549 (siRNA targeting PSGL-1) + activated PLTs; 5, HuvECs+A549 + activated PLTs + P-selectin antibody. ${ }^{*} \mathrm{P}<0.05,{ }^{* *} \mathrm{P}<0.01$ as compared with HuvECs+A549.

was $13 \%$ and the rolling rate of A549 was decreased by only $11 \%$ after the co-culture of HuvECs and A549 cells (Fig. 5).
After the RNAi targeting P-selectin specific ligand PSGL-1, adhesion efficiency was $27 \%$ and the rolling rate of A549 was $76 \%$ after the co-culture of HuvECs, A549 and activated PLTs. After the co-culture of HuvECs, A549 after RNAi targeting PSGL-1 and activated PLTs, the specific P-selectin antibody was employed to block the binding site of PSGL-1. Then the adhesion efficiency was $22 \%$ and the rolling rate of A549 was reduced by $20 \%$ (Fig. 5).

$m R N A$ expression of integrin $\alpha 3$, integrin $\alpha 5$, integrin $\beta 1$, ICAM-1 and VCAM-1 are up-regulated after the co-culture of HuvECs, A549 and activated PLTs. The RT-PCR results suggested that the mRNA levels of integrin $\alpha 3$, integrin $\alpha 5$, integrin $\beta 1$, ICAM- 1 and VCAM-1 after the co-culture of HuvECs, A549 and activated PLTs were significantly increased compared with that after the co-culture of HuvECs and A549 cells $(\mathrm{P}<0.05)$ (Fig. 6).

Protein expression of integrin $\alpha 3$, integrin $\alpha 5$, integrin $\beta 1$, ICAM-1 and VCAM-1 are up-regulated after the co-culture of HuvECs, A549 and activated PLTs. The results of western blot analysis showed that the protein levels of integrin $\alpha 3$, integrin $\alpha 5$, integrin $\beta 1$, ICAM- 1 and VCAM- 1 the co-culture of HuvECs, A549 and activated PLTs were significantly increased compared with that after the co-culture of HuvECs and A549 cells $(\mathrm{P}<0.05)$ (Fig. 7).

\section{Discussion}

Lung cancer is a severe disease and its morbidity and death rates are gradually increasing. Lung cancer is becoming the leading cause of cancer-related deaths. Distant hematogenous metastasis results in poor prognosis and the deaths of lung cancer patients $(1,2)$. Tumor hematogenous metastasis is a complex multistep process including separation and leakage from the primary
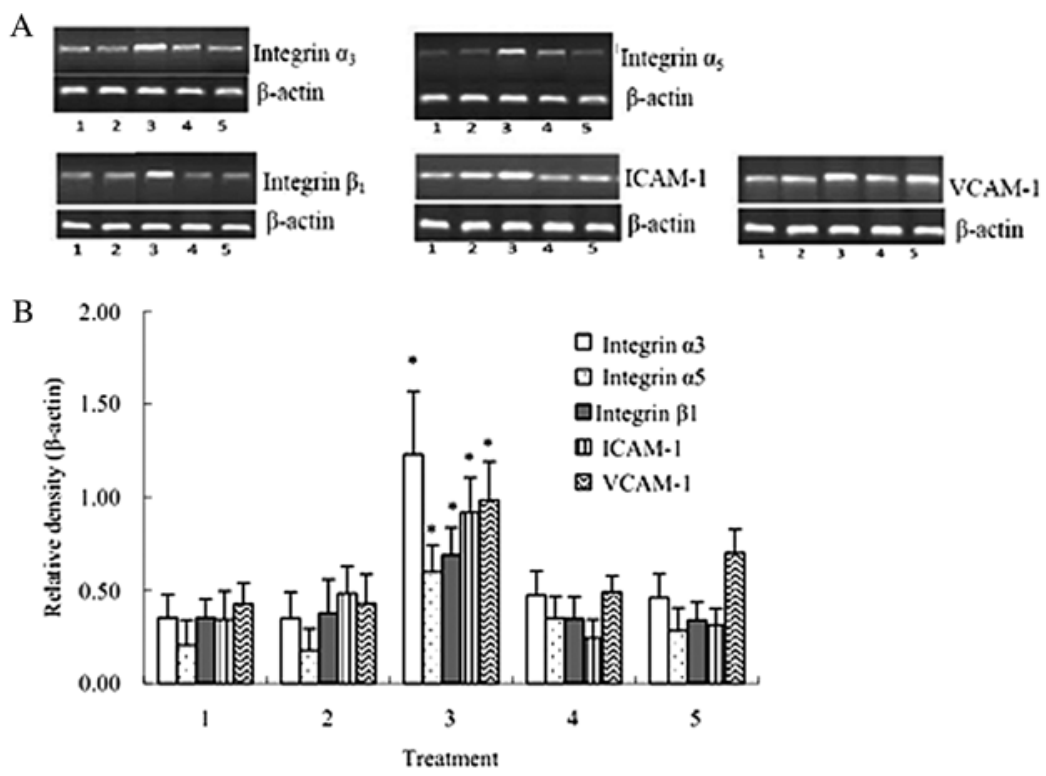

Figure 6. mRNA expression of integrin $\alpha 3$, integrin $\alpha 5$, integrin $\beta 1$, ICAM- 1 and VCAM- $1( \pm \mathrm{SD}, \mathrm{n}=3)$. mRNA levels of integrin $\alpha 3$, integrin $\alpha 5$, integrin $\beta 1$, ICAM-1 and VCAM-1 after the co-culture of HuvECs, A549 and activated PLTs were significantly increased compared with that after the co-culture of HuvECs and A549. 1, HuvECs+A549; 2, HuvECs+A549 + inactivated PLTs; 3, HuvECs+A549 + activated PLTs; 4, HuvECs+A549 (siRNA targeting PSGL-1) + activated PLTs; 5, HuvECs+A549 + activated PLTs + P-selectin antibody. ${ }^{*}$ P $<0.05$ as compared with HuvECs+A549. 
A

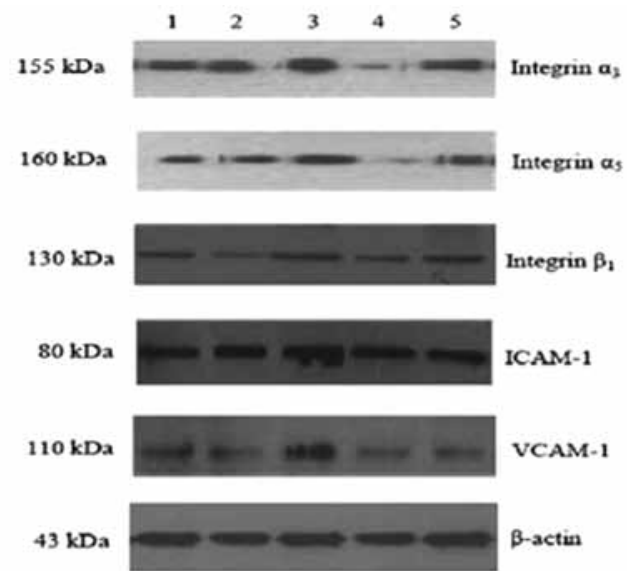

B

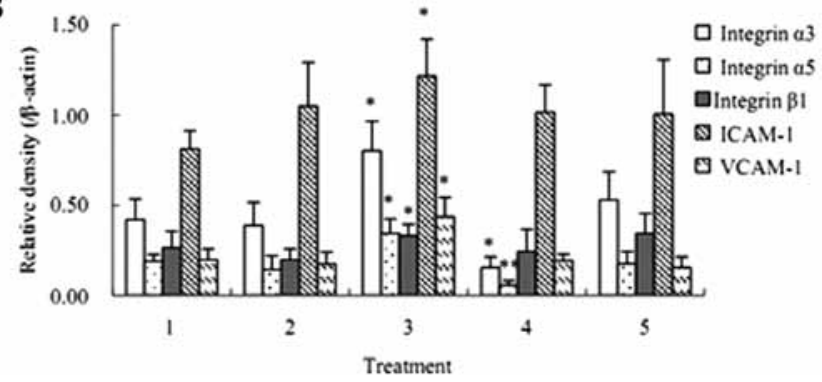

Figure 7. Protein expression of integrin $\alpha 3$, integrin $\alpha 5$, integrin $\beta 1$, ICAM-1 and VCAM-1 (mean $\pm S D, n=3$ ). The protein levels of integrin $\alpha 3$, integrin $\alpha 5$, integrin $\beta 1$, ICAM- 1 and VCAM- 1 after the co-culture of HuvECs, A549 and activated PLTs were significantly up-regulated compared with that after the co-culture of HuvECs and A549. 1, HuvECs+A549; 2, HuvECs+A549 + inactivated PLTs; 3, HuvECs+A549 + activated PLTs; 4, HuvECs+A549 (siRNA targeting PSGL-1) + activated PLTs; 5, HuvECs+A549 + activated PLTs + P-selectin antibody. ${ }^{*} \mathrm{P}<0.05,{ }^{* *} \mathrm{P}<0.01$ as compared with HuvECs+A549.

tumor, promotion of angiogenesis and entry into the circulatory system, escape from attack by circulatory blood and immune factors, adhesion with endothelial cells in target organs, and eventual passage through the vascular endothelium and formation of metastasis via distant planting $(3,6-8)$. At present, more and more studies have confirmed that PLT activation in the circulation is relevant to hematogenous metastasis of a variety of malignant tumors $(4,5,9)$, while anti-platelet factor may inhibit tumor metastasis $(10,11)$. However, the mechanism has not been clarified thoroughly.

Platelet aggregation and increased expression of active molecules are main characteristics of platelet activation. P-selectin is an important cell adhesion molecule that normally exits in platelet $\alpha$ particle. An absence and persistently low expression were found in the resting state. P-selectin can be rapidly expressed at the surface of PLTs by membrane fusion effect when PLTs are activated. Therefore, P-selectin is commonly selected as a marker for PLT activation $(12,13)$. Simanek et al confirmed that a high blood platelet count is an important and independent factor for venous thrombosis in cancer patients in the clinic (14). The blood platelet count was reported to be a valuable prediction index for the prognosis of a variety of cancers including malignant melanoma, renal carcinoma, gastric carcinoma, and breast carcinoma $(4,15,16)$. Iwasaki et al found that a high blood platelet count was a significant independent factor for lung cancer postoperative metastasis (17). Dymicka-Piekarska et al suggested that soluble $\mathrm{P}$-selectin is associated with malignant invasion and metastasis of colon carcinoma (18).

In the present study, we found that the expression of $\mathrm{P}$-selectin in lung adenocarcinoma was higher than that in paracancerous tissue, distant tissue and lung squamous cell carcinoma. Meanwhile, significant increased blood platelet count and P-selectin were also found in the peripheral blood of NSCLC patients. Compared with patients in stage I+II and III, the increase in PLTs in the patients in stage IV was significant $(\mathrm{P}<0.05)$. Furthermore, the FACS results showed that $\mathrm{P}$-selectin activation in lung adenocarcinoma was significantly enhanced compared with that in the healthy control and lung squamous cell carcinoma, suggesting a correlation between PLT activation, P-selectin expression and hematogenous metastasis of NSCLC particularly lung adenocarcinoma.

The cultured human lung adenocarcinoma cell line A549 interacted with PLTs to form activated PLT-lung adenocarcinoma cell complexes. It has been confirmed that adhesion between cancer cells and PLTs is associated with the metastatic potential. The adhesion molecules that may interact with tumor cells mainly include GPIb-IX-V, GPIIb/IIIa and P-selectin (19-21). However, the detail of their interactions particularly in the presence of blood flow in vivo is not fully understood $(22,23)$. In the present study we found that the rolling rate of A549 cells on the surface of vascular endothelial cells was significantly decreased after co-culture of activated PLTs and A549 in a flow state. Alternatively, the effect of inactivated PLTs, P-selectin antibody or siRNA targeting P-selectin ligand PSGL-1 on lung adenocarcinoma cells was significantly attenuated, which was consistent with the study of Borsig et al (24). They found that heparin and its derivatives efficiently suppressed the metastasis of various tumors via reducing the combination of tumor cells and P-selectin in transplanted tumors in vivo (25). These results suggest that the activated PLT-lung adenocarcinoma cell complexes affect the capture of lung adenocarcinoma cells on the surface of vascular endothelium via $\mathrm{P}$-selectin mediation.

Tumor cell capture and adhesion is a key process for hematogenous metastasis (26), which is regulated by a series of cellular adhesion molecules including cadherins, selectin, integrin, immunoglobulin family and CD44 (27). Generally, adhesion capacity of individual tumor cells to vascular endothelial cells is comparatively lower. Combined with the erosion blood flow, the adhesion capacity is further attenuated. McCarty et al observed that PLTs support the capture, rolling and stable adhesion of colon carcinoma in a blood flow condition (28), which was similar to our study. Integrin was found to play a crucial role in regulating stable cellular adhesion $(29,30)$. It was reported that in inflammatory reactions P-selectin promotes leucocytic adhesion and migration via initiating the activation of leucocyte integrin (31). In lung cancer, major histological types express integrin $\beta 1$. Integrin $\alpha 2, \alpha 3, \alpha 6, \alpha \mathrm{V}$ and $\beta 1$ were mainly expressed in lung squamous cell carcinoma and smallcell carcinoma, and integrin $\alpha 3 \beta 1$ and $\alpha 5 \beta 1$ were strongly expressed in lung adenocarcinoma $(32,33)$. Our results showed that mRNA and protein levels of lung cancer cell membrane P-selectin ligand glycoprotein PSGL-1, integrin $\alpha 3, \alpha$ and $\beta 1$, endothelial cell adhesion molecule ICAM-1, VCAM-1 gene 
were increased in different degree after the co-culture of A549 cells, activated PLTs and HuvECs.

In summary, the activation of circulatory PLTs affected the hematogenous metastasis of NSCLC particularly lung adenocarcinoma. The activated PLT-lung cancer cell complexes protected the cancer cells from mechanical injury by blood flow and escaped attack by the immune system. In addition, the complexes up-regulated the expression of integrin $\alpha 3$, $\alpha 5, \beta 1$ and endothelial cell adhesion molecules ICAM-1 and VCAM-1, thereby promoting the capture of NSCLC cells on the surface of vascular endothelial cells, which may be one of the mechanisms for hematogenous metastasis of lung cancer due to PLT activation. Importantly, anti-PLT activation was found to play a potential role in suppressing tumor metastasis (34-36). Further study on the mechanism concerning the PLT promotion of hematogenous metastasis of tumors will aid in the search for specific target drugs to suppress tumor metastasis and to provide a new direction for individualized treatment.

\section{References}

1. Baldwin DR: Lung cancer: investigation and staging. Medicine 36: 155-161, 2008.

2. Jemal A, Siegel R, Ward E, et al: Cancer statistics, 2008. CA Cancer J Clin 58: 71-96, 2008.

3. Leber MF and Efferth T: Molecular principles of cancer invasion and metastasis (Review). Int J Oncol 34: 881-895, 2009.

4. Borsig L: The role of platelet activation in tumor metastasis. Expert Rev Anticancer Ther 8: 1247-1255, 2008.

5. Tsuruo T and Fujita N: Platelet aggregation in the formation of tumor metastasis. Proc Jpn Acad Ser B Phys Biol Sci 84: 189-198, 2008.

6. Chambers AF, Naumov GN, Varghese HJ, et al: Critical steps in hematogenous metastasis: an overview. Surg Oncol Clin N Am 10: 243-255, 2001.

7. Geiger TR and Peeper DS: Metastasis mechanisms. Biochim Biophys Acta 1796: 293-308, 2009.

8. Joyce JA and Pollard JW: Microenvironmental regulation of metastasis. Nat Rev Cancer 9: 239-252, 2008.

9. Noble S and Pasi J: Epidemiology and pathophysiology of cancerassociated thrombosis. Br J Cancer 13: S2-S9, 2008.

10. Amirkhosravi A, Mousa SA, Amaya M, et al: Inhibition of tumor cell-induced platelet aggregation and lung metastasis by the oral GPIIb/IIIa antagonist XV454. Thromb Haemost 90: 549-554, 2003.

11. Sierko E and Wojtukiewicz MZ: Inhibition of platelet function: does it offer a chance of better cancer progression control? Semin Thromb Hemost 33: 712-721, 2007.

12. Ludwig RJ, Schön MP and Boehncke WH: P-selectin: a common therapeutic target for cardiovascular disorders, inflammation and tumour metastasis. Expert Opin Ther Targets 11: 1103-1117, 2007.

13. Massaguer A, Engel $\mathrm{P}$, Tovar V, et al: Characterization of platelet and soluble-porcine P-selectin (CD62P). Vet Immunol Immunopathol 96: 169-181, 2003.

14. Simanek R, Vormittag R, Ay C, et al: High platelet count associated with venous thromboembolism in cancer patients: results from the Vienna Cancer and Thrombosis Study (CATS). J Thromb Haemost 8: 114-120, 2010.

15. Verheul HM and Pinedo HM: The importance of platelet counts and their contents in cancer. Clin Cancer Res 9: 3219-3221, 2003.
16. Ikeda $M$, Furukawa $H$, Imamura $H$, et al: Poor prognosis associated with thrombocytosis in patients with gastric cancer. Ann Surg Oncol 9: 287-291, 2002.

17. Iwasaki A, Hamanaka W, Harnada T, et al: Significance of platelet counts in patients who underwent surgical treatment for lung metastasis. Int Surg 92: 103-109, 2007.

18. Dymicka-Piekarska V, Matowicka-Karna J, Gryko M, et al: Relationship between soluble P-selectin and inflammatory factors (interleukin-6 and C-reactive protein) in colorectal cancer. Thromb Res 120: 585-590, 2007.

19. Erpenbeck L and Schön MP: Deadly allies: the fatal interplay between platelets and metastasizing cancer cells. Blood 115: 3427-3436, 2010

20. Jurasz P, Alonso-Escolano D and Radomski MW: Platelet-cancer interactions: mechanisms and pharmacology of tumour cellinduced platelet aggregation. Br J Pharmacol 143: 819-826, 2004.

21. Trikha M, Zhou Z, Timar J, et al: Multiple roles for platelet GPIIb/IIIa and $\alpha v \beta 3$ integrins in tumor growth, angiogenesis, and metastasis. Cancer Res 62: 2824-2833, 2002.

22. Fuchs B, Budde U, Schulz A, et al: Flow-based measurements of von Willebrand factor (VWF) function: binding to collagen and platelet adhesion under physiological shear rate. Thromb Res 125: 239-245, 2010.

23. Cominetti MR, Martin AC, Ribeiro JU, et al: Inhibition of platelets and tumor cell adhesion by the disintegrin domain of human ADAM9 to collagen I under dynamic flow conditions. Biochimie 91: 1045-1052, 2009.

24. Borsig L, Wong R and Hynes RO: Synergistic effects of L- and P-selectin in facilitating tumor metastasis can involve non-mucin ligands and implicate leukocytes as enhancers of metastasis. Proc Natl Acad Sci USA 99: 2193-2198, 2002.

25. Baumann K, Kowalczyk D, Gutjahr T, et al: Sulfated and non-sulfated glycopeptide recognition domains of P-selectin glycoprotein ligand 1 and their binding to P- and E-selectin. Angew Chem Int Ed Engl 48: 3174-3178, 2009.

26. Iiizumi M, Mohinta S, Bandyopadhyay S and Watabe K: Tumorendothelial cell interactions: therapeutic potential. Microvasc Res 74: 114-120, 2007.

27. Skubitz AP: Adhesion molecules. Cancer Treat Res 107: 305-329, 2002.

28. McCarty OJ, Mousa SA, Bray PF and Konstantopoulos K: Immobilized platelets support human colon carcinoma cell tethering, rolling, and firm adhesion under dynamic flow conditions. Blood 96: 1789-1797, 2000.

29. Ley K: cell adhesion under flow. Microcirculation 16: 1-2, 2009.

30. Arnaout MA, Goodman SL and Xiong JP: Structure and mechanics of integrin-based cell adhesion. Cell Biology 19: 495-507, 2007.

31. Wang HB, Wang JT, Zhang L, et al: P-selectin primes leukocyte integrin activation during inflammation. Nat Immunol 8: 882-892, 2007.

32. Gogali A, Charalabopoulos $\mathrm{K}$ and Constantopoulos S: Integrin receptors in primary lung cancer. Exp Oncol 26: 106-110, 2004.

33. Syrigos KN, Katirtzoglou N, Kotteas E and Harrington K: Adhesion molecules in lung cancer: implications in the pathogenesis and management. Curr Pharm Des 14: 2173-2183, 2008.

34. Barthel SR, Gavino JD, Descheny L and Dimitroff CJ: Targeting selectins and selectin ligands in inflammation and cancer. Expert Opin Ther Targets 11: 1473-1491, 2007.

35. Borsig L: Antimetastatic activities of modified heparins: selectin inhibition by heparin attenuates metastasis. Semin Thromb Hemost 33: 540-546, 2007.

36. Marathe DD, Buffone A Jr, Chandrasekaran EV, et al: Fluorinated per-acetylated GalNAc metabolically alters glycan structures on leukocyte PSGL-1 and reduces cell binding to selectins. Blood 115: 1303-1312, 2010. 